INPLASY

PROTOCOL

To cite: Rivera et al. Brain Arteriovenous Malformation Models for Clinical Practice: Protocol for a Scoping Review. Inplasy protocol 2020100064. doi:

10.37766/inplasy2020.10.0064

Received: 19 October 2020

Published: 19 October 2020

Corresponding author:

Rodrigo Rivera

rodrigo.rivera@etu.unilim.fr

Author Affiliation:

PhD Diploma: Innovation

Technologique et

Thérapeutique. Universite de Limoges

Support: No financial support.

Review Stage at time of this submission: Data analysis.

Conflicts of interest:

No conflicts of interest to declare.

\section{Brain Arteriovenous Malformation Models for Clinical Practice: Protocol for a Scoping Review}

Rivera, R1; Cruz, JP2; Merino-Osorio, C3; Rouchaud, A4; Mounayer, $\mathrm{C}^{5}$.

Review question / Objective: Which are the in vivo or in vitro brain Arteriovenous malformation models and their use? Condition being studied: Large animals like swine, sheep or dogs have been used frequently as their vessel size make them more suitable for neurointerventional testing. Other models like small animals have also been used, but mostly for molecular studies, genetic expression or radiosurgery treatment. In vitro models have been an alternative, thus they have not been able to reach the validation as the animal models, mainly because of the vessel size within the nidus and the complex configuration of bAVMs that are difficult to recreate in a 3D or 2D models. Finally, computer simulated models have been tested as virtual replicas for a better understanding of these malformations. Hence, the aim of this scoping review is to comprehensively map and summarize the current knowledge of the in vivo or in vitro models of bAVMs.

INPLASY registration number: This protocol was registered with the International Platform of Registered Systematic Review and Meta-Analysis Protocols (INPLASY) on 19 October 2020 and was last updated on 19 October 2020 (registration number INPLASY2020100064).

\section{INTRODUCTION}

Review question / Objective: Which are the in vivo or in vitro brain Arteriovenous malformation models and their use?
Rationale: Brain arteriovenous malformations (bAVM) are lesions composed of complex abnormal connections between arteries and veins. They don't follow the characteristic vascular structure, so arteries and veins 
are connected by a tangled vessel configuration known as the "nidus". Although they are rare, with a prevalence that has been estimated in 15-18 per 100.000 adults, bAVM can produce severe clinical consequences and neurological impairment when they rupture and lead to intracerebral hemorrhage (ICH). Many of the therapeutic options for bAVMs have been tested in several models before human use, like the use of new materials or the effects of radiosurgery. Because of their complex structure, it has been challenging to adequately replicate a bAVM in an in vivo or in vitro model. Moreover, there are other special features that are difficult to replicate, like flow, arteriovenous shunt, or the biological interaction between the malformation and the surrounding brain. Which are the in vivo or in vivo that have been use for bAVM? There is a need in summarizing and classifying the existing models for bAVMs.

Condition being studied: Large animals like swine, sheep or dogs have been used frequently as their vessel size make them more suitable for neurointerventional testing. Other models like small animals have also been used, but mostly for molecular studies, genetic expression or radiosurgery treatment. In vitro models have been an alternative, thus they have not been able to reach the validation as the animal models, mainly because of the vessel size within the nidus and the complex configuration of bAVMs that are difficult to recreate in a 3D or 2D models. Finally, computer simulated models have been tested as virtual replicas for a better understanding of these malformations. Hence, the aim of this scoping review is to comprehensively map and summarize the current knowledge of the in vivo or in vitro models of bAVMs.

\section{METHODS}

Search strategy: To identify relevant studies, two investigators (RR and CMO) will search the literature based on the three stages as recommended by the JBI. First a MEDLINE PubMed search will be performed using keyword MeSH terms associated to: 'Arteriovenous Malformations', 'Intracranial Arteriovenous Malformations', 'Animal Models', 'Biological Models', 'Anatomic Models' 'Endovascular Procedures', 'Neurosurgical Procedures', 'Radiotherapy' and 'Neurosurgery'. Second, the key words in the relevant papers of the first search will be identified and included in a second electronic search (limited to available databases in our institutions). The following biomedical databases will be consulted: PubMed (NCBI), CINHAL Plus with Full Text (EBSCO) Embase, Epistemonikos, Clinical Trials and Cochrane Library (Free access from the Chilean ministry of health website). Language filters (English and Spanish) will be applied. All kind of experimental articles will be considered, research articles or conference abstracts. Moreover, the grey literature will be search using Opengrey. Third, a handmade research of the reference list of relevant papers will be made. A bibliography software to manage all references (Mendeley 1.19.4) will be used. The final search will be made in May 5th 2020.

Participant or population: The population under study will be brain arteriovenous malformation's models.

Intervention: In this review we will consider articles in which bAVM models are used for therapeutic, anatomical or biological studies.

Comparator: Exposure will not be compared.

Study designs to be included: This review will consider all type of experimetal articles, original studies, conference abstracts and trial protocols.

Eligibility criteria: We will consider all experimental studies that includes bAVM models used for therapeutic, anatomical or biological studies.

Information sources: The search will be made in electronic sources: PubMed (NCBI), CINAHL, Embase, Epistemonikos, Clinical Trials, OpenGrey, Cochrane Library. 
Main outcome(s): The main outcome of this review will be all the described models (in vivo or in vivo) used for brain arteriovenous malformations. They should include their nature, type, use and description.

Data management: Once the search is complete by two authors, all identified elements will be managed using COVIDENCE(r) software for selection, elimination of duplicates and full text review. This process will also be carried out by two independent and blinded authors. If there are discrepancies in election, these will be discussed and in case of persistence a third author will decide. We will use the include rather than the exclude criteria. This stage will be reported using the PRISMA flow chart. Data will be extracted using an Excel spreadsheet by two authors and one doing quality control. Extracted data will be Author names, year of publication, title and DOI, Country of main author, objective of study, nature of model, type of model, use of model, technical specifications and description of use.

Quality assessment / Risk of bias analysis: One author will be performing quality assessment, by choosing randomly $10 \%$ of articles in the extraction process, In previous process, there will be double author blinded checking and a third one deliberating when in doubt.

Strategy of data synthesis: The obtained data will be organized and summarized by two researchers (RR, JPC). A third author will make quality control with random selected information (CMO). This will be an iterative process that will vary in relation to bibliographic findings. We will use the PRISMA flow chart for Scoping reviews which will be delivered by COVIDENCE Software. Selected and excluded articles will be reported. Results will be presented in tables and iconographic summary.

Subgroup analysis: We will not performed a subgroup analysis
Sensibility analysis: It is not within the objectives of this study to perform a sensibility analysis.

Language: All articles in English or Spanish.

Country(ies) involved: Chile and France.

Keywords: Scoping Review, Arteriovenous Malformation, Models.

Dissemination plans: Once the overview is completed, it will be edited in the format of a medical scientific journal, to which it will be submitted for publication.

Contributions of each author:

Author 1 - Rodrigo Rivera - Lead Author, Developed general idea and protocol, wrote protocol and future manuscript.

Author 2 - Juan Pablo Cruz - co-author, participate in the development of general idea, review protocol and future manuscript.

Author 3 - Catalina Merino-Osorio - coauthor, participate in the development of general idea, review protocol and future manuscript.

Author 4 - Aymeric Rouchaud - co-author, participate in the development of general idea, review protocol, commentaries and feedback.

Author 5 - Charbel Mounayer - co-author, participate in the development of general idea, review protocol, commentaries and feedback. 